\title{
Newton-Cartan gravity and torsion
}

\author{
Eric Bergshoeff, ${ }^{a}$ Athanasios Chatzistavrakidis, ${ }^{a, b}$ Luca Romano $^{a}$ and Jan Rosseel ${ }^{c}$ \\ ${ }^{a}$ Van Swinderen Institute for Particle Physics and Gravity, University of Groningen, \\ Nijenborgh 4, 9747 AG Groningen, The Netherlands \\ ${ }^{b}$ Division of Theoretical Physics, Rudjer Bošković Institute, \\ Bijenička 54, 10000 Zagreb, Croatia \\ ${ }^{c}$ Faculty of Physics, University of Vienna, \\ Boltzmanngasse 5, A-1090, Vienna, Austria \\ E-mail: e.a.bergshoeff@rug.nl, a.chatzistavrakidis@gmail.com, \\ lucaromano2607@gmail.com, rosseelj@gmail.com
}

ABSTRACT: We compare the gauging of the Bargmann algebra, for the case of arbitrary torsion, with the result that one obtains from a null-reduction of General Relativity. Whereas the two procedures lead to the same result for Newton-Cartan geometry with arbitrary torsion, the null-reduction of the Einstein equations necessarily leads to Newton-Cartan gravity with zero torsion. We show, for three space-time dimensions, how Newton-Cartan gravity with arbitrary torsion can be obtained by starting from a Schrödinger field theory with dynamical exponent $z=2$ for a complex compensating scalar and next coupling this field theory to a $z=2$ Schrödinger geometry with arbitrary torsion. The latter theory can be obtained from either a gauging of the Schrödinger algebra, for arbitrary torsion, or from a null-reduction of conformal gravity.

Keywords: Classical Theories of Gravity, Space-Time Symmetries

ARXIV EPRINT: 1708.05414 


\section{Contents}

1 Introduction 1

2 Gauging the Bargmann algebra with arbitrary torsion $\quad 4$

$\begin{array}{lll}3 & \text { The null-reduction of general relativity } & 6\end{array}$

4 Gauging the $z=2$ Schrödinger algebra with arbitrary torsion $\quad 10$

5 The null-reduction of conformal gravity 12

$\begin{array}{lll}6 & \text { NC gravity with arbitrary torsion } & 14\end{array}$

$\begin{array}{llr}7 & \text { Conclusions } & 18\end{array}$

\section{Introduction}

Usually, when discussing Newton-Cartan (NC) geometry and gravity, Newtonian causality is incorporated by imposing that the space-time manifold admits a one-form $\tau_{\mu}$, called the time-like Vierbein, ${ }^{1}$ whose curl is constrained to vanish. The vanishing of the curl of $\tau_{\mu}$ is often referred to as the 'zero torsion condition' and implies the existence of an absolute time in the space-time geometry. Indeed, using the one-form $\tau_{\mu}$ one can define the time difference $T$ between two events as

$$
T=\int_{\mathcal{C}} \mathrm{d} x^{\mu} \tau_{\mu}
$$

where $\mathcal{C}$ is a path connecting the two events. The zero torsion condition implies that the time difference $T$ is independent of the path $\mathcal{C}$ connecting the two events and can thus indeed be identified with an absolute time. Alternatively, the zero torsion condition allows one to express $\tau_{\mu}$ as the derivative of a single scalar field $\tau(x)$ :

$$
\partial_{\mu} \tau_{\nu}-\partial_{\nu} \tau_{\mu}=0 \quad \Rightarrow \quad \tau_{\mu}=\partial_{\mu} \tau
$$

Since then

$$
T=\int_{\mathcal{C}} \mathrm{d} x^{\mu} \tau_{\mu}=\int_{\mathcal{C}} \mathrm{d} \tau
$$

one sees that the absolute time $t$ can be identified with this function $\tau(x)$ :

$$
\tau(x)=t \quad \Rightarrow \quad \tau_{\mu}=\delta_{\mu}^{0} .
$$

\footnotetext{
${ }^{1}$ Most of this paper applies to any space-time dimension. We will therefore from now on use the word Vielbein instead of Vierbein.
} 
The zero-torsion condition (1.2) is sufficient but not necessary to obtain a causal nonrelativistic geometry. Indeed, Frobenius' theorem states that a necessary and sufficient condition for the space-time to admit a foliation in a time flow orthogonal to Riemannian space-like leaves (and thus obey non-relativistic causality), is the so-called hypersurface orthogonality condition

$$
\tau_{[\mu} \partial_{\nu} \tau_{\rho]}=0
$$

that can be equivalently written as

$$
\tau_{a b} \equiv e_{a}{ }^{\mu} e_{b}{ }^{\nu} \tau_{\mu \nu}=0, \quad \tau_{\mu \nu}=\partial_{[\mu} \tau_{\nu]},
$$

where $e_{a}{ }^{\mu}$ is the projective inverse of the spatial Vielbein $e_{\mu}{ }^{a}$, with $\mu=0,1, \cdots d-1$ and $a=1,2, \cdots d-1$, see eq. (2.12). Note that in this case the time difference between two space-like leaves depends on the path between the two leaves, i.e. there is no well-defined notion of an absolute time on which all observers agree.

The condition (1.6), also called the twistless-torsional condition, was first encountered in the context of Lifshitz holography when studying the coupling of Newton-Cartan gravity to the Conformal Field Theory (CFT) at the boundary [1]. Twistless-torsional NewtonCartan geometry has also been applied in studies of the Quantum Hall Effect [2] and has been encountered in a large $c$ expansion of General Relativity [3]. Note that it is not surprising that the more general twistless-torsional condition (1.6) was found in the context of CFTs. The zero torsion condition (1.2) is simply not allowed within a CFT since it is not invariant under space-time-dependent dilatations $\delta \tau_{\mu} \sim \Lambda_{D}(x) \tau_{\mu}$. Instead, the condition (1.6) is invariant under space-time-dependent dilatations due to the relation $e_{a}{ }^{\mu} \tau_{\mu}=0$, see eq. (2.12).

In the presence of local dilatation symmetry, one can define a conformal, i.e. dilatationcovariant, torsion as

$$
\tau_{\mu \nu}^{C} \equiv \partial_{[\mu} \tau_{\nu]}-2 b_{[\mu} \tau_{\nu]},
$$

where $b_{\mu}$ is the gauge field of dilatations, i.e. it transforms under dilatations as $\delta b_{\mu}=\partial_{\mu} \Lambda_{D}$. The twistless-torsional condition (1.6) can then also be equivalently restated as

$$
\tau_{\mu \nu}^{C}=0
$$

Indeed, by taking the space/space projection of this equation, one obtains (1.6):

$$
\tau_{a b}^{C} \equiv e_{a}{ }^{\mu} e_{b}{ }^{\nu} \tau_{\mu \nu}^{C}=\tau_{a b}=0 .
$$

The space/time projection of $\tau_{\mu \nu}^{C}=0$ does not lead to an extra constraint on $\tau_{\mu \nu}$, but can instead be used to solve for the spatial components of $b_{\mu}$ :

$$
\tau_{0 a}^{C} \equiv \tau^{\mu} e_{a}{ }^{\nu} \tau_{\mu \nu}^{C}=0 \quad \Rightarrow \quad b_{a} \equiv e_{a}{ }^{\mu} b_{\mu}=-\tau_{0 a} .
$$

where we used that $\tau^{\mu} \tau_{\mu}=1$, see eq. (2.12).

In this paper, we will be interested in considering non-relativistic geometry, both in the absence and presence of conformal symmetries, in the case of arbitrary torsion, i.e. 
when the zero torsion or twistless-torsional conditions no longer hold. At first sight, it seems strange to consider the case of arbitrary torsion since causality is lost in this case. However, in condensed matter applications, one often considers gravity not as a dynamical theory but as background fields for determining the response of the system to a geometrical force and for defining a non-relativistic energy and momentum flux. ${ }^{2}$ It was pointed out a long time ago in the seminal paper by Luttinger [4] that to describe thermal transport in a resistive medium one needs to consider an auxiliary gravitational field $\psi(x)$ that couples to the energy and is defined by [5]

$$
\tau_{\mu}=e^{\psi(x)} \delta_{\mu}^{0}
$$

corresponding to the case of twistless torsion. Later, it was pointed out that, for describing other properties as well, one also needs to introduce the other components of $\tau_{\mu}$ that couple to the energy current. This leads to a non-relativistic energy-momentum tensor with no restrictions and an un-restricted $\tau_{\mu}$ describing arbitrary torsion [5]. For other applications of torsion in condensed matter, see $[6,7] .{ }^{3}$ To avoid confusion, we will reserve the word 'geometry' if we only consider the background fields and their symmetries whereas we will talk about 'gravity' if these background fields satisfy dynamical equations of motion.

In this paper, we will construct by two complementary techniques, gauging and nullreduction, the extension of $\mathrm{NC}$ geometry and its non-relativistic conformal extension, Schrödinger geometry with dynamical exponent $z=2$, to the case of arbitrary torsion, i.e. $\tau_{\mu \nu} \neq 0$ for NC geometry and $\tau_{a b} \neq 0$ for Schrödinger geometry, see table 1. Furthermore, applying a different technique thereby making use of the obtained results on Schrödinger geometry with arbitrary torsion, we will construct the extension of NC gravity to the case of arbitrary torsion, in three space-time dimensions. Note that in the conformal case we will always impose that $\tau_{0 a}^{C}=0$, i.e. the minimal torsion case is twistless-torsional, in agreement with the fact that the zero torsion condition is incompatible with dilatation symmetry. As explained above, $\tau_{0 a}^{C}=0$ does not lead to a constraint on $\tau_{\mu \nu}$. Rather it is a so-called conventional constraint, that can be used to solve for $b_{a}$, see eq. (1.10). For earlier discussions of Newton-Cartan geometry with torsion and null-reductions, see [8-12].

This paper is organized as follows. In section 2 we will apply the gauging technique to the Bargmann algebra in $d$ space-time dimensions. In particular, we will construct the transformation rules of the independent fields and the expressions of the dependent spinconnections of $\mathrm{NC}$ geometry for the case of arbitrary torsion. In section 3 we derive the same results from an off-shell, meaning we do not reduce the equations of motion, null-reduction of General Relativity in $d+1$ space-time dimensions. We point out that performing a null-reduction of the equations of motion as well we obtain the equations of motion of $\mathrm{NC}$ gravity with zero torsion thereby reproducing the result of [9]. We point out that the zero torsion condition is related to the invariance under central charge transformations that necessarily follows from the null-reduction. To obtain NC gravity with arbitrary torsion,

\footnotetext{
${ }^{2}$ This applies to the microscopic theory. Gravitational fields can occur dynamically in an effective field theory description.

${ }^{3}$ In [7] non-zero expressions for the spatial torsion, i.e. the curl of the spatial Vielbein, and for the curl of the central charge gauge field are considered as well. We will not consider this more general situation here.
} 


\begin{tabular}{|c|c|c|c|}
\hline geometric constraint & Newton-Cartan & geometric constraint & Schrödinger \\
\hline$\tau_{0 a} \neq 0, \tau_{a b} \neq 0$ & arbitrary torsion & $\tau_{a b} \neq 0$ & arbitrary torsion \\
$\tau_{0 a} \neq 0, \tau_{a b}=0$ & twistless-torsional & $\tau_{a b}=0$ & twistless-torsional \\
$\tau_{0 a}=0, \tau_{a b}=0$ & zero torsion & - & - \\
\hline
\end{tabular}

Table 1. Newton-Cartan and Schrödinger geometry with torsion.

we will first in the next two sections repeat the calculations of sections 2 and 3 but now for the minimal conformal extension of the Bargmann algebra, i.e. the Schrödinger algebra, and for conformal gravity except that we do not consider the equations of motion in this case. To be precise, in section 4 we will gauge the $z=2$ Schrödinger algebra and obtain the transformation rules of $z=2$ Schrödinger geometry for arbitrary torsion together with the expressions of the dependent gauge fields. Next, in section 5 , we obtain the same results by performing a null-reduction of conformal gravity in $d+1$ space-time dimensions. In section 6 , we use these results to construct three-dimensional NC gravity with arbitrary torsion by starting from a $z=2$ Schrödinger Field Theory (SFT) for a complex compensating scalar, coupling it to the Schrödinger geometry with arbitrary torsion we constructed in sections 4 and 5 and gauge-fixing the dilatations and central charge transformations. We give our comments in the Conclusions.

\section{Gauging the Bargmann algebra with arbitrary torsion}

Our starting point is the $d$-dimensional Bargmann algebra whose non-zero commutators are given by

$$
\begin{array}{rlrl}
{\left[J_{a b}, J_{c d}\right]} & =4 \delta_{[a[c} J_{d] b]}, & {\left[J_{a b}, P_{c}\right]} & =-2 \delta_{c[a} P_{b]}, \\
{\left[J_{a b}, G_{c}\right]} & =-2 \delta_{c[a} G_{b]}, & {\left[G_{a}, H\right]} & =-P_{a}, \\
{\left[G_{a}, P_{b}\right]} & =-\delta_{a b} M, &
\end{array}
$$

where

$$
\left\{H, P_{a}, J_{a b}, G_{a}, M\right\}
$$

are the generators corresponding to time translations, spatial translations, spatial rotations, Galilean boosts and central charge transformations, respectively. Note that the $M$-generator has the dimension of a mass and that for $M=0$ the Bargmann algebra reduces to the Galilei algebra. The gauging of the Bargmann algebra for zero torsion has been considered in [13]. In this section we will extend this gauging to the case of arbitrary torsion, see also [12].

The gauge fields corresponding to the generators (2.2) are given by

$$
\left\{\tau_{\mu}, e_{\mu}^{a}, \omega_{\mu}^{a b}, \omega_{\mu}^{a}, m_{\mu}\right\}
$$

respectively. Under general coordinate transformations, they transform as covariant vectors. Under the spatial rotations, Galilean boosts and central charge transformations, with 
parameters $\left\{\lambda^{a}{ }_{b}, \lambda^{a}, \sigma\right\}$, respectively, the gauge fields $\left\{\tau_{\mu}, e_{\mu}{ }^{a}, m_{\mu}\right\}$ that will remain independent, see below, transform according to the structure constants of the Bargmann algebra, i.e.:

$$
\begin{aligned}
\delta \tau_{\mu} & =0, \\
\delta e_{\mu}{ }^{a} & =\lambda^{a}{ }_{b} e_{\mu}{ }^{b}+\lambda^{a} \tau_{\mu}, \\
\delta m_{\mu} & =\partial_{\mu} \sigma+\lambda^{a} e_{\mu a} .
\end{aligned}
$$

These independent fields and their transformation rules then define $\mathrm{NC}$ geometry in the presence of arbitrary torsion, i.e. $\tau_{\mu \nu} \neq 0$.

Now that we have arbitrary torsion, we can modify the usual conventional constraints that can be solved for the spin-connection fields $\omega_{\mu}^{a b}$ and $\omega_{\mu}^{a}$ such that these spinconnections receive torsion contributions. We choose the following conventional constraints that are justified by the null-reduction of General Relativity that we will perform in the next section:

$$
\begin{aligned}
R_{\mu \nu}\left(P^{a}\right)+2 \tau_{[\mu}^{a} m_{\nu]} & =0, \\
R_{\mu \nu}(M)-2 \tau_{0[\mu} m_{\nu]} & =0,
\end{aligned}
$$

with $\tau_{0 \nu} \equiv \tau^{\mu} \tau_{\mu \nu}, \tau_{a \nu} \equiv e_{a}{ }^{\mu} \tau_{\mu \nu}$ and with the curvatures $R_{\mu \nu}\left(P^{a}\right)$ and $R_{\mu \nu}(M)$ given by expressions that follow from the structure constants of the Bargmann algebra:

$$
\begin{aligned}
R_{\mu \nu}\left(P^{a}\right) & =2 \partial_{[\mu} e_{\nu]}^{a}-2 \omega_{[\mu}^{a b} e_{\nu] b}-2 \omega_{[\mu}^{a} \tau_{\nu]}, \\
R_{\mu \nu}(M) & =2 \partial_{[\mu} m_{\nu]}-2 \omega_{[\mu}^{a} e_{\nu] a} .
\end{aligned}
$$

Explicitly, the expressions for the torsionful spin-connections that follow from the constraints (2.5) and (2.6) are given by ${ }^{4}$

$$
\begin{aligned}
\omega_{\mu}{ }^{a b}(\tau, e, m) & =\stackrel{\circ}{\omega}_{\mu}^{a b}(\tau, e, m)-m_{\mu} \tau^{a b}, \\
\omega_{\mu}{ }^{a}(\tau, e, m) & =\dot{\leftrightarrow}_{\mu}^{a}(\tau, e, m)+m_{\mu} \tau_{0}{ }^{a},
\end{aligned}
$$

where the space/space and space/time components of the torsion are given by

$$
\tau_{a b}=e_{a}^{\mu} e_{b}^{\nu} \partial_{[\mu} \tau_{\nu]}, \quad \tau_{0 a}=\tau^{\mu} e^{\nu}{ }_{a} \partial_{[\mu} \tau_{\nu]}
$$

and where $\dot{\omega}_{\mu}^{a b}(\tau, e, m)$ and $\dot{\circ}_{\mu}^{a}(\tau, e, m)$ are the torsion-free Newton-Cartan spinconnections given by

$$
\begin{aligned}
\stackrel{\omega}{\omega}_{\mu}^{a b}(\tau, e, m) & =e_{\mu c} e^{\rho a} e^{\sigma b} \partial_{[\rho} e_{\sigma]}^{c}-e^{\nu a} \partial_{[\mu} e_{\nu]}^{b}+e^{\nu b} \partial_{[\mu} e_{\nu]}^{a}-\tau_{\mu} e^{\rho a} e^{\sigma b} \partial_{[\rho} m_{\sigma]} \\
\dot{\omega}_{\mu}^{a}(\tau, e, m) & =\tau^{\nu} \partial_{[\mu} e_{\nu]}^{a}+e_{\mu}{ }^{c} e^{\rho a} \tau^{\sigma} \partial_{[\rho} e_{\sigma] c}+e^{\nu a} \partial_{[\mu} m_{\nu]}+\tau_{\mu} \tau^{\rho} e^{\sigma a} \partial_{[\rho} m_{\sigma]} .
\end{aligned}
$$

The expressions for $\dot{\omega}_{\mu}^{a b}$ and $\dot{\omega}_{\mu}^{a}$ are the solutions of the constraints (2.5) and (2.6) for zero torsion, i.e. $\tau_{\mu \nu}=0$. Note that the solutions (2.10) and (2.11) contain the fields

\footnotetext{
${ }^{4}$ Note that from now on the spin-connections are dependent fields. In cases, when confusion could arise, we will indicate the explicit dependence.
} 
$\tau^{\mu}$ and $e^{\mu}{ }_{a}$ that are defined by the following projective invertibility relations

$$
\begin{aligned}
& e^{\mu}{ }_{a} e_{\nu}{ }^{a}=\delta_{\nu}^{\mu}-\tau^{\mu} \tau_{\nu}, \quad e^{\mu}{ }_{a} e_{\mu}{ }^{b}=\delta_{b}^{a}, \\
& \tau^{\mu} \tau_{\mu}=1, \quad e^{\mu}{ }_{a} \tau_{\mu}=0, \quad \tau^{\mu} e_{\mu}{ }^{a}=0 .
\end{aligned}
$$

It is important to note that the dependent torsion-free spin-connections $\stackrel{\circ}{\omega}_{\mu}^{a b}(\tau, e, m)$ and $\dot{\omega}_{\mu}{ }^{a}(\tau, e, m)$, due to the arbitrary torsion, no longer transform according to the Bargmann algebra. In particular, from eqs. (2.10) and (2.11) it follows that their transformation rules under Galilean boosts contain extra torsion terms given by

$$
\begin{aligned}
\Delta \dot{\omega}_{\mu}{ }^{a b} & =\lambda^{c} e_{\mu c} \tau^{a b}+2 \lambda^{[a} e^{|\rho| b]} \tau_{\mu \rho}, \\
\Delta \dot{\omega}_{\mu}{ }^{a} & =-\lambda^{a} e_{\mu}{ }^{b} \tau_{0 b}-\lambda^{b} e_{\mu b} \tau_{0}{ }^{a} .
\end{aligned}
$$

Correspondingly, the curvatures corresponding to these spin-connections that transform covariantly under Galilean boosts contain extra torsion contributions and are given by

$$
\begin{aligned}
R_{\mu \nu}\left(J^{a b}\right) & =2 \partial_{[\mu} \stackrel{\circ}{\omega}_{\nu]}^{a b}-2 \stackrel{\circ}{\omega}_{[\mu}^{a c} \stackrel{\circ}{\omega}_{\nu] c}^{b}-2 \stackrel{\circ}{\omega}_{[\mu}^{c} e_{\nu] c} \tau^{a b}-4 \stackrel{\circ}{\omega}_{[\mu}{ }^{[a} e^{|\rho| b]} \tau_{\nu] \rho}, \\
R_{\mu \nu}\left(G^{a}\right) & =2 \partial_{[\mu} \stackrel{\circ}{\omega}_{\nu]}^{a}-2 \stackrel{\circ}{\omega}_{[\mu}^{a b} \stackrel{\circ}{\omega}_{\nu] b}+2 \stackrel{\circ}{\omega}_{[\mu}^{a} e_{\nu]}{ }^{b} \tau_{0 b}+2 \stackrel{\circ}{\omega}_{[\mu}^{b} e_{\nu] b} \tau_{0}{ }^{a} .
\end{aligned}
$$

These are the curvatures that naturally appear in the next section when we perform a nullreduction of the equations of motion of General Relativity, see eq. (3.17). Note that there is an arbitrariness in the definition of these curvatures in the sense that one can always move around torsion terms in or outside the spin-connections. In that sense the above curvatures are defined modulo $D \tau$ and $\tau^{2}$ terms. The specific definition we use naturally follows from the null-reduction in the next section.

\section{The null-reduction of general relativity}

In this section we re-obtain the results on NC geometry with arbitrary torsion obtained in the previous section by performing a dimensional reduction of General Relativity (GR) from $d+1$ to $d$ space-time dimensions along a null-direction $[8,9]$. We show that in this way one obtains the same transformation rules and the same expressions for the dependent spin-connections as before. Next, we point out that, after going on-shell, the equations of motion reduce to those of $\mathrm{NC}$ gravity with zero torsion $[8,9]$.

Our starting point is General Relativity in $d+1$ dimensions in the second order formalism, where the single independent field is the Vielbein $\hat{e}_{M}{ }^{A}$. Here and in the following, hatted fields are $(d+1)$-dimensional and unhatted ones will denote $d$-dimensional fields after dimensional reduction. Furthermore, capital indices take $d+1$ values, with $M$ being a curved and $A$ a flat index. The Einstein-Hilbert action in $d+1$ space-time dimensions is given by

$$
S_{\mathrm{GR}}^{(d+1)}=-\frac{1}{2 \kappa} \int \mathrm{d}^{d+1} x \hat{e} \hat{e}^{M}{ }_{A} \hat{e}^{N}{ }_{B} \hat{R}_{M N}{ }^{A B}(\hat{\omega}(\hat{e})),
$$

where $\kappa$ is the gravitational coupling constant and $\hat{e}$ is the determinant of the Vielbein. The inverse Vielbein satisfies the usual relations

$$
\hat{e}^{M}{ }_{A} \hat{e}_{M}{ }^{B}=\delta_{A}^{B}, \quad \hat{e}^{M}{ }_{A} \hat{e}_{N}{ }^{A}=\delta_{N}^{M} .
$$


The spin-connection is a dependent field, given in terms of the vielbein as

$$
\hat{\omega}_{M}^{B A}(\hat{e})=2 \hat{e}^{N[A} \partial_{[M} \hat{e}_{N]}^{B]}-\hat{e}^{N[A} \hat{e}^{B] P} \hat{e}_{M C} \partial_{N} \hat{e}_{P}^{C},
$$

while the curvature tensor is given by

$$
\hat{R}_{M N}^{A B}(\hat{\omega}(\hat{e}))=2 \partial_{[M} \hat{\omega}_{N]}^{A B}-2 \hat{\omega}_{[M}^{A C} \hat{\omega}_{N] C}{ }^{B} .
$$

Under infinitesinal general coordinate transformations, with parameter $\zeta^{M}$ and local Lorentz transformations, with parameter $\lambda^{A}{ }_{B}$, the Vielbein transforms as

$$
\delta \hat{e}_{M}{ }^{A}=\zeta^{N} \partial_{N} \hat{e}_{M}{ }^{A}+\partial_{M} \zeta^{N} \hat{e}_{N}{ }^{A}+\lambda^{A}{ }_{B} \hat{e}_{M}{ }^{B} .
$$

In order to dimensionally reduce the transformation rules along a null-direction, we assume the existence of a null Killing vector $\xi=\xi^{M} \partial_{M}$ for the metric $\hat{g}_{M N} \equiv \hat{e}_{M}{ }^{A} \hat{e}_{N}{ }^{B} \eta_{A B}$, i.e.

$$
\mathcal{L}_{\xi} \hat{g}_{M N}=0 \quad \text { and } \quad \xi^{2}=0 .
$$

Without loss of generality, we may choose adapted coordinates $x^{M}=\left\{x^{\mu}, v\right\}$, with $\mu$ taking $d$ values, and take the Killing vector to be $\xi=\xi^{v} \partial_{v}$. Then the Killing equation implies that the metric is $v$-independent, i.e. $\partial_{v} \hat{g}_{M N}=0$, while the null condition implies the following constraint on the metric: ${ }^{5}$

$$
\hat{g}_{v v}=0 .
$$

A suitable reduction Ansatz for the Vielbein should be consistent with this constraint on the metric. Such an Ansatz was discussed in [9], and we repeat it below in a formalism suited to our purposes.

First, we split the $(d+1)$-dimensional tangent space indices as $A=\{a,+,-\}$, where the index $a$ is purely spatial and takes $d-1$ values, while \pm denote null directions. Then the Minkowski metric components are $\eta_{a b}=\delta_{a b}$ and $\eta_{+-}=1$. The reduction Ansatz is specified upon choosing the inverse Vielbein $\hat{e}^{M}+$ to be proportional to the null Killing vector $\xi=\xi^{v} \partial_{v}$. A consistent parametrization is

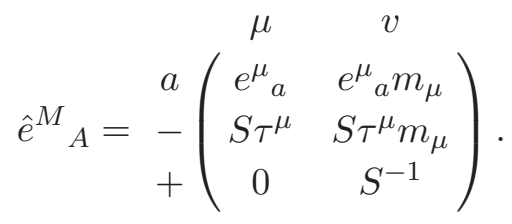

The scalar $S$ is a compensating one and can be gauge-fixed as we will see shortly.

Given the expression (3.8) for the inverse Vielbein, the Vielbein itself is given by

$$
\hat{e}_{M}^{A}={ }_{v}^{\mu}\left(\begin{array}{ccc}
e^{a} & - & + \\
e^{a} & S^{-1} \tau_{\mu} & -S m_{\mu} \\
0 & 0 & S
\end{array}\right) .
$$

\footnotetext{
${ }^{5}$ Due to this constraint, we are not allowed to perform the null-reduction in the action but only in the transformation rules and equations of motion [9].
} 
To avoid confusion, recall that the index $a$ takes one value less than the index $\mu$; thus the above matrices are both square although in block form this is not manifest.

Note that the Ansatz (3.9) has two zeros. The zero in the second column, $\hat{e}_{v}{ }^{-}=0$, is due to the existence of the null Killing vector $\xi=\xi^{v} \partial_{v}$ :

$$
\xi^{2}=\xi^{v} \xi^{v} \hat{g}_{v v}=0 \quad \Rightarrow \quad \hat{g}_{v v}=\hat{e}_{v}{ }^{A} \hat{e}_{v}{ }^{B} \eta_{A B}=0 \quad \Rightarrow \quad \hat{e}_{v}{ }^{-}=0 .
$$

On the other hand, the zero in the first column, $\hat{e}_{v}{ }^{a}=0$, implies that the Lorentz transformations with parameters $\lambda^{a}+$ are gauge-fixed. We are thus left over with $\lambda^{a}{ }_{b}, \lambda^{a}{ }_{-}$, that we will call $\lambda^{a} \equiv \lambda^{a}{ }_{-}$, and $\lambda^{+}{ }_{+}=-\lambda^{-}{ }_{-}$, that we will call $\lambda$. The latter can be gauge-fixed by imposing $S=1$. For some purposes, especially when we discuss the conformal case, it is convenient to only perform this gauge-fixing at a later stage, so we will momentarily keep $S$.

A simple computation reveals that the invertibility relations (3.2), after substitution of the reduction Ansatz, precisely reproduce the projective invertibility relations (2.12) encountered when gauging the Bargmann algebra provided we identify $\left\{\tau_{\mu}, e_{\mu}{ }^{a}\right\}$ as the timelike and spatial Vielbein of $\mathrm{NC}$ gravity, respectively.

Starting from the transformation rule $(3.5)$ of the $(d+1)$-dimensional Vielbein, we derive the following transformations of the lower-dimensional fields:

$$
\begin{aligned}
\delta \tau_{\mu} & =0, \\
\delta e_{\mu}{ }^{a} & =\lambda^{a}{ }_{b} e_{\mu}{ }^{b}+S^{-1} \lambda^{a} \tau_{\mu}, \\
\delta m_{\mu} & =-\partial_{\mu} \zeta^{v}-S^{-1} \lambda_{a} e_{\mu}{ }^{a}, \\
\delta S & =\lambda S,
\end{aligned}
$$

where $\zeta^{v}$ denotes the component of the parameter of $(d+1)$-dimensional diffeomorphisms, along the compact $v$-direction. Next, fixing the Lorentz transformations with parameter $\lambda$ by setting $S=1$ and defining $\sigma:=-\zeta^{v}$ we precisely obtain the transformation rules (2.4) of Newton-Cartan geometry in $d$ dimensions provided we identify $m_{\mu}$ as the central charge gauge field associated to the central charge generator of the Bargmann algebra. Note that we have not imposed any constraint on the torsion, i.e. $\tau_{\mu \nu}=\partial_{[\mu} \tau_{\nu]} \neq 0$.

We next consider the null-reduction of the spin-connection given in (3.3). Inserting the Vielbein Ansatz (3.9) with $S=1$ into (3.3) we obtain the following expressions for the different components:

$$
\begin{array}{rlrl}
\hat{\omega}_{\mu}^{a b}(\hat{e}) & \equiv \omega_{\mu}^{a b}(\tau, e, m)=\stackrel{\circ}{\mu}_{\mu}^{a b}(e, \tau, m)-m_{\mu} \tau^{a b}, & & \\
\hat{\omega}_{\mu}{ }^{a+}(\hat{e}) & \equiv \omega_{\mu}^{a}(\tau, e, m)=\dot{\circ}_{\mu}^{a}(e, \tau, m)+m_{\mu} \tau_{0}^{a}, & & \hat{\omega}_{v}{ }^{a+}(\hat{e})=-\tau_{0}{ }^{a}, \\
\hat{\omega}_{v}{ }^{a b}(\hat{e}) & =\tau^{a b}, & \hat{\omega}_{v}{ }^{a-}(\hat{e})=0, \\
\hat{\omega}_{\mu}{ }^{a-}(\hat{e}) & =-\tau_{\mu} \tau_{0}{ }^{a}-e_{\mu}{ }^{b} \tau_{b}{ }^{a}, & & \hat{\omega}_{v}{ }^{-+}(\hat{e})=0, \\
\hat{\omega}_{\mu}{ }^{-+}(\hat{e}) & =-e_{\mu}{ }^{b} \tau_{0 b}, &
\end{array}
$$

where $\stackrel{\circ}{\omega}_{\mu}^{a b}(e, \tau, m)$ and $\stackrel{\circ}{\omega}_{\mu}^{a}(e, \tau, m)$ are the torsion-free Newton-Cartan spin-connections given in eqs. (2.10) and (2.11). Note that the first two lines precisely reproduce the expressions for the torsionful spin-connections of $\mathrm{NC}$ gravity given in eqs. (2.8) of the previous section. 
At this point, we have re-produced using the complementary null-reduction technique the results on NC geometry with arbitrary torsion obtained in the previous section. To calculate the equations of motion after null-reduction, we first need to calculate the components of the higher-dimensional Ricci tensor with flat indices:

$$
\hat{R}_{A B}(\hat{\omega}(\hat{e}))=\hat{e}^{M}{ }_{C} \hat{e}^{N}{ }_{A} \hat{R}_{M N}{ }^{C}{ }_{B}(\hat{\omega}(\hat{e})) .
$$

Substituting the reduction Ansatz (3.9), with $S=1$, into (3.16) we find the following expressions for the Ricci tensor components:

$$
\begin{aligned}
\hat{R}_{++} & =-\tau^{a b} \tau_{a b}, \\
\hat{R}_{+-} & =-D_{a} \tau_{0}{ }^{a}+2 \tau_{0}{ }^{a} \tau_{0 a}, \\
\hat{R}_{--} & =-R_{0 a}\left(G^{a}\right) \\
\hat{R}_{+a} & =D_{b} \tau^{b}{ }_{a}-2 \tau_{0}{ }^{b} \tau_{b a}, \\
\hat{R}_{-a} & =-R_{0 b}\left(J^{b}{ }_{a}\right)-D_{0} \tau_{0 a}, \\
\hat{R}_{a b} & =R_{c a}\left(J^{c}{ }_{b}\right)-2 D_{a} \tau_{0 b}+D_{0} \tau_{a b}+2 \tau_{0 a} \tau_{0 b},
\end{aligned}
$$

where the lower-dimensional curvatures $R(J)$ and $R(G)$ are defined in eq. (2.14) and where the covariant derivatives on $\tau_{0}{ }^{a}$ and $\tau^{a b}$ are given by

$$
\begin{aligned}
& D_{\mu} \tau_{0}{ }^{a}=\partial_{\mu} \tau_{0}{ }^{a}-\stackrel{\leftrightarrow}{\omega}_{\mu}{ }^{a b} \tau_{0 b}+\stackrel{\circ}{\omega}_{\mu}{ }^{b} \tau_{b}{ }^{a}, \\
& D_{\mu} \tau^{a b}=\partial_{\mu} \tau^{a b}-\stackrel{\circ}{\omega}_{\mu}{ }^{a}{ }_{c} \tau^{c b}-\stackrel{\circ}{\omega}_{\mu}{ }^{b}{ }_{c} \tau^{a c} .
\end{aligned}
$$

Using the Bianchi identity for $\tau_{\mu \nu}$ in the form

$$
D_{0} \tau_{a b}=D_{a} \tau_{0 b}-D_{b} \tau_{0 a},
$$

we can rewrite the Ricci tensor components $\hat{R}_{a b}$ in a manifestly symmetric form as follows:

$$
\hat{R}_{a b}=R_{c a}\left(J_{b}^{c}\right)-2 D_{(a} \tau_{|0| b)}+2 \tau_{0 a} \tau_{0 b} .
$$

We first consider the Ricci tensor components that contain the curvatures $R(J)$ and/or $R(G)$. They lead to the following set of equations of motion:

$$
R_{0 a}\left(G^{a}\right)=0, \quad R_{c \bar{a}}\left(J_{b}^{c}\right)-2 D_{(\bar{a}} \tau_{|0| b)}+2 \tau_{0 \bar{a}} \tau_{0 b}=0,
$$

where in the last equation we collected two field equations into one by using an index $\bar{a}=(a, 0)$. At first sight, it looks like this first set of equations of motion defines NC gravity with arbitrary torsion. However, the other set of equations, obtained by putting $\hat{R}_{++}, \hat{R}_{+-}$and $\hat{R}_{+a}$ to zero, cannot be ignored and they constrain the torsion. For instance, the equation $\hat{R}_{++}=0$ implies $\tau_{a b}=0$ while the equation $\hat{R}_{+-}=0$ implies, with a proper choice of boundary conditions, $\tau_{0 a}=0$. Since the first set of equations of motion transforms to the second one under Galilean boosts, it is not consistent to leave out the second set of equations of motion in the hope of obtaining $\mathrm{NC}$ equations of motion with arbitrary torsion. Together, they imply zero torsion and, after substituting this back into (3.21), one obtains the equations of motion corresponding to NC gravity with zero torsion [9].

$$
R_{0 a}\left(G^{a}\right)=0, \quad R_{c 0}{ }^{c} b(J)=0, \quad R_{c a}^{c}{ }_{b}(J)=0 .
$$




\section{Gauging the $z=2$ Schrödinger algebra with arbitrary torsion}

In this section we extend the gauging of the so-called $z=2$ Schrödinger algebra with twistless torsion as performed in [15] to the case of arbitrary torsion, i.e. $\tau_{a b} \neq 0$. Our starting point is the $z=2$ Schrödinger algebra which is the minimal conformal extension, with dynamical exponent $z=2$, of the $d$-dimensional Bargmann algebra whose commutation relations were given in eq. (2.1). To this end we add the additional generators $D$ and $K$ corresponding to dilatations and special conformal transformations with gauge fields $b_{\mu}$ and $f_{\mu}$, respectively. The additional non-zero commutation relations with respect to the Bargmann algebra are given by

$$
\begin{aligned}
{[D, H] } & =-2 H, & {[H, K] } & =D, \\
{[D, K] } & =2 K, & {\left[K, P_{a}\right] } & =-G_{a}, \\
{\left[D, P_{a}\right] } & =-P_{a}, & {\left[D, G_{a}\right] } & =G_{a} .
\end{aligned}
$$

This leads us to the following complete set of covariant one-form gauge fields:

$$
\left\{e_{\mu}^{a}, \tau_{\mu}, \omega_{\mu}^{a b}, \omega_{\mu}^{a}, b_{\mu}, f_{\mu}, m_{\mu}\right\} .
$$

Only the subset $\left\{\tau_{\mu}, e_{\mu}{ }^{a}, m_{\mu}, b_{0}\right\}$, with $b_{0} \equiv \tau^{\mu} b_{\mu}$, will remain independent gauge fields. Following the structure constants of the Schrödinger algebra these independent gauge fields transform under the Bargmann symmetries and the additional dilatations, with parameter $\lambda_{D}$, and special conformal transformations, with parameter $\lambda_{K}$, as follows:

$$
\begin{aligned}
\delta \tau_{\mu} & =2 \lambda_{D} \tau_{\mu}, \\
\delta e_{\mu}{ }^{a} & =\lambda^{a}{ }_{b} e_{\mu}{ }^{b}+\lambda^{a} \tau_{\mu}+\lambda_{D} e_{\mu}{ }^{a}, \\
\delta m_{\mu} & =\partial_{\mu} \sigma+\lambda^{a} e_{\mu a}, \\
\delta b_{0} & =\partial_{0} \lambda_{D}+\lambda_{K}-\lambda^{a} e_{a}{ }^{\mu} b_{\mu} .
\end{aligned}
$$

We now impose the following first set of conventional curvature constraints: ${ }^{6}$

$$
\begin{aligned}
\mathcal{R}_{0 a}(H) & =0, \\
\mathcal{R}_{\mu \nu}{ }^{a}(P)+2 \tau^{C a}{ }_{[\mu} m_{\nu]} & =0, \\
\mathcal{R}_{\mu \nu}(M) & =0 .
\end{aligned}
$$

We have used here the following curvatures whose expressions follow from the structure constants of the Schrödinger algebra:

$$
\begin{aligned}
\mathcal{R}_{\mu \nu}(H) & =2 \partial_{[\mu} \tau_{\nu]}-4 b_{[\mu} \tau_{\nu]}, \\
\mathcal{R}_{\mu \nu}{ }^{a}(P) & =2 \partial_{[\mu} e_{\nu]}{ }^{a}-2 \omega_{[\mu}{ }^{a b} e_{\nu] b}-2 \omega_{[\mu}{ }^{a} \tau_{\nu]}-2 b_{[\mu} e_{\nu]}{ }^{a}, \\
\mathcal{R}_{\mu \nu}(M) & =2 \partial_{[\mu} m_{\nu]}-2{\omega_{[\mu}}^{a} e_{\nu] a} .
\end{aligned}
$$

\footnotetext{
${ }^{6}$ We indicate the Schrödinger curvatures with a script $\mathcal{R}$. Note that, in contrast to [15], we do not impose that $\mathcal{R}_{a b}(H)=0$, i.e. we have arbitrary torsion: $\frac{1}{2} \mathcal{R}_{a b}(H)=\tau_{a b}^{C}=\tau_{a b} \neq 0$. We have chosen the second conventional constraint such that it gives the same torsionful rotational spin-connection that follows from the null-reduction that we will perform in the next section.
} 
The conventional constraints (4.3) allow us to solve for the spatial components of $b_{\mu}$ and of the spin-connection fields $\omega_{\mu}^{a b}$ and $\omega_{\mu}^{a}$ as follows ${ }^{7}$

$$
\begin{aligned}
b_{a} & =-\tau_{0 a}, \\
\omega_{\mu}^{a b}(e, \tau, m, b) & =\stackrel{\circ}{\omega}_{\mu}^{a b}(e, \tau, m, b)-m_{\mu} \tau^{a b}, \\
\omega_{\mu}^{a}(e, \tau, m, b) & =\stackrel{\leftrightarrow}{\omega}_{\mu}^{a}(e, \tau, m, b),
\end{aligned}
$$

where the torsionless Schrödinger spin-connections, i.e. the part with $\tau_{a b}=0$, are related to the torsionless Newton-Cartan spin-connections defined in eqs. (2.10) and (2.11) as follows: ${ }^{8}$

$$
\begin{aligned}
& \stackrel{\circ}{\mu}_{\mu}^{a b}(e, \tau, m, b)=\dot{\circ}_{\mu}^{a b}(e, \tau, m)+2 e_{\mu}{ }^{[a} b^{b]}, \\
& \stackrel{\circ}{\omega}_{\mu}^{a}(e, \tau, m, b)=\dot{\omega}_{\mu}{ }^{a}(e, \tau, m)+e_{\mu}{ }^{a} b_{0} .
\end{aligned}
$$

In a second step, to solve for the gauge field $f_{\mu}$, we impose the following second set of conventional constraints:

$$
\begin{aligned}
\mathcal{R}_{a 0}(D)+\mathcal{R}_{a b}{ }^{b}(G)-\frac{1}{2 d} m_{a} \mathcal{R}_{b c}{ }^{b c}(J) & =0, \\
\mathcal{R}_{0 a}{ }^{a}(G)-\frac{1}{2 d} m_{0} \mathcal{R}_{a b}{ }^{a b}(J) & =0,
\end{aligned}
$$

where the expressions for the curvatures are given by

$$
\begin{aligned}
& \mathcal{R}_{\mu \nu}^{a b}(J)=2 \partial_{[\mu} \stackrel{\circ}{\omega}_{\nu]}^{a b}-2 \stackrel{\circ}{\omega}_{[\mu}^{c a} \stackrel{\circ}{\omega}_{\nu]}^{b} c-2 \stackrel{\circ}{\omega}_{[\mu}^{c} e_{\nu] c} \tau^{a b}+4 \stackrel{\circ}{\omega}_{[\mu}^{[a} \tau^{b] c} e_{\nu] c}-4 \dot{\circ}_{[\mu}^{c} e_{\nu]}{ }^{[a} \tau_{c}^{b]}, \\
& \mathcal{R}_{\mu \nu}{ }^{a}(G)=2 \partial_{[\mu} \stackrel{\circ}{\nu}_{\nu]}^{a}+2 \dot{\omega}_{[\mu}{ }^{b} \stackrel{\circ}{\omega}_{\nu]}^{a} b-2 \dot{\circ}_{[\mu}^{a} b_{\nu]}-2 f_{[\mu} e_{\nu]}^{a}, \\
& \mathcal{R}_{\mu \nu}(D)=2 \partial_{[\mu} b_{\nu]}-2 f_{[\mu} \tau_{\nu]}+2 \dot{\omega}_{[\mu}^{b} e_{\nu]}^{a} \tau_{a b} \text {. }
\end{aligned}
$$

Note that these curvatures, save the one corresponding to $G^{a}$, contain extra torsion contributions that render them covariant under Galilean boosts. This second set of conventional constraints is chosen such that it precisely reproduces the same expression for $f_{\mu}$ that we will derive in the next section by a null-reduction of conformal gravity:

$$
\begin{aligned}
& f_{a}=\frac{1}{d-1} \mathcal{R}_{a 0}^{\prime}(D)+\frac{1}{d-1} \mathcal{R}_{a b}^{\prime b}(G)-\frac{1}{2 d(d-1)} m_{a} \mathcal{R}_{b c}{ }^{b c}(J) \\
& f_{0}=\frac{1}{d-1} \mathcal{R}_{0 a}^{\prime a}(G)-\frac{1}{2 d(d-1)} m_{0} \mathcal{R}_{a b}{ }^{a b}(J)
\end{aligned}
$$

The prime indicates that in the corresponding curvature the term with $f_{\mu}$ has been omitted.

This finishes our discussion of the gauging of the $z=2$ Schrödinger algebra.

\footnotetext{
${ }^{7}$ The only notational difference with respect to [15] is that in that paper the projective inverse of $\tau_{\mu}$ is denoted as $v^{\mu}$ and it is related to the one we use here by $v^{\mu}=-\tau^{\mu}$.

${ }^{8}$ Note that we commit some abuse of notation here, by using the same symbol $\stackrel{\dot{\omega}}{\text { for }} \dot{\omega}_{\mu}^{a b}(e, \tau, m)$, $\dot{\omega}_{\mu}^{a}(e, \tau, m)$ and $\dot{\omega}_{\mu}{ }^{a b}(e, \tau, m, b), \stackrel{\circ}{\omega}_{\mu}^{a}(e, \tau, m, b)$. For the rest of this paper, $\dot{\omega}_{\mu}{ }^{a b}$ and $\dot{\omega}_{\mu}{ }^{a}$ will always refer to $\dot{\omega}_{\mu}^{a b}(e, \tau, m, b), \stackrel{\circ}{\omega}_{\mu}^{a}(e, \tau, m, b)$.
} 


\section{The null-reduction of conformal gravity}

In this section we re-obtain the results on $z=2$ Schrödinger geometry with arbitrary torsion obtained in the previous section by performing a dimensional reduction of conformal gravity from $d+1$ to $d$ space-time dimensions along a null direction. We show that in this way one obtains the same transformation rules and the same expressions for the dependent spin-connections and special conformal gauge fields as before.

Our starting point is conformal gravity in $d+1$ dimensions. Recall that the relativistic conformal algebra appends new generators to the translations and Lorentz transformations of the Poincaré algebra, namely dilatations and special conformal transformations. When the algebra is gauged, the dilatations give rise to a gauge field $\hat{b}_{M}$ with associated gauge parameter $\lambda_{D}$ while the special conformal transformations are assigned a gauge field $\hat{f}_{M}{ }^{A}$ and gauge parameters $\lambda_{K}^{A} \cdot{ }^{9}$ Thus the full set of gauge fields is

$$
\left\{\hat{e}_{M}^{A}, \hat{\omega}_{M}^{A B}, \hat{b}_{M}, \hat{f}_{M}^{A}\right\} .
$$

It turns out that after imposing conventional constraints the spin-connection and special conformal gauge fields become dependent. The transformation rules of the independent Vielbein and dilatation gauge field are given by

$$
\begin{aligned}
\delta \hat{e}_{M}{ }^{A} & =\lambda^{A}{ }_{B} \hat{e}_{M}{ }^{B}+\lambda_{D} \hat{e}_{M}{ }^{A}, \\
\delta \hat{b}_{M} & =\partial_{M} \lambda_{D}+\lambda_{K}^{A} \hat{e}_{M A} .
\end{aligned}
$$

Both gauge fields transform as covariant vectors under general coordinate transformations. Note that the dilatation gauge field transforms with a shift under the special conformal transformations and therefore can be gauged away by fixing the $K$-transformations. The expressions for the dependent spin-connections and special conformal gauge fields are given by

$$
\begin{aligned}
\hat{\omega}_{M}{ }^{A B}(\hat{e}, \hat{b}) & =\hat{\omega}_{M}{ }^{A B}(\hat{e})+2 \hat{e}_{M}{ }^{[A} \hat{e}^{B] N} \hat{b}_{N}, \\
\hat{f}_{M}{ }^{A}(\hat{e}, \hat{b}) & =-\frac{1}{d-1} \hat{\mathcal{R}}_{M}^{\prime}{ }^{A}+\frac{1}{2 d(d-1)} \hat{e}_{M}{ }^{A} \hat{\mathcal{R}}^{\prime},
\end{aligned}
$$

where $\hat{\mathcal{R}}_{M N}{ }^{A B}$ is the Lorentz curvature of the conformal algebra and

$$
\hat{\mathcal{R}}_{M}^{\prime}{ }^{A}=\hat{\mathcal{R}}_{M N}^{\prime A B} \hat{e}^{N}{ }_{B}, \quad \hat{\mathcal{R}}^{\prime}=\hat{e}^{M}{ }_{A} \hat{\mathcal{R}}_{M}^{\prime}{ }^{A} .
$$

The prime indicates that in the corresponding curvature the term with $\hat{f}_{M}^{A}$ has been omitted.

Using the same reduction Ansatz as in the NC case and splitting $\hat{b}_{M}=\left(b_{\mu}, b_{v}\right)$, we obtain the following transformation rules for the lower-dimensional fields:

$$
\begin{aligned}
\delta \tau_{\mu} & =2 \lambda_{D} \tau_{\mu}, \\
\delta e_{\mu}{ }^{a} & =\lambda^{a}{ }_{b} e_{\mu}{ }^{b}+S^{-1} \lambda^{a} \tau_{\mu}+\lambda_{D} e_{\mu}{ }^{a}, \\
\delta m_{\mu} & =-\partial_{\mu} \zeta^{v}-S^{-1} \lambda_{a} e_{\mu}{ }^{a}, \\
\delta b_{\mu} & =\partial_{\mu} \lambda_{D}+\lambda_{K}^{a} e_{\mu a}+\lambda_{K}^{+} S^{-1} \tau_{\mu}-\lambda_{K}^{-} S m_{\mu}, \\
\delta b_{v} & =\lambda_{K}^{-} S \\
\delta S & =\left(\lambda+\lambda_{D}\right) S .
\end{aligned}
$$

\footnotetext{
${ }^{9}$ Like in the Poincaré case we denote fields in $d+1$ dimensions with a hat.
} 
From the last transformation rule it follows that gauge-fixing $S=1$ leads this time to a compensating Lorentz transformation with parameter

$$
\lambda_{\text {comp }}=-\lambda_{D}
$$

The $S=1$ gauge-fixing is not sufficient to end up with the transformation rules of $z=2$ Schrödinger geometry as given in the previous section. The reason for this is that the nullreduction leads to as many $K$-transformations as components of $\hat{b}_{M}$ while in Schrödinger geometry we have only a single $K$-transformation. This is related to the fact that the $z=2$ Schrödinger algebra cannot be embedded into a higher-dimensional conformal algebra like the Bargmann algebra can be embedded into a higher-dimensional Poincaré algebra. In order to obtain the same symmetries as $z=2$ Schrödinger geometry we need to impose a constraint that reduces the $d+1 K$-transformations to the single one corresponding to the Schrödinger algebra. To achieve this, we first gauge-fix $b_{v}=0$ which fixes $\lambda_{K}^{-}=0$. To gauge-fix another $d-1 K$-transformations we impose by hand the following constraint

$$
\mathcal{R}_{0 a}(H)=0 \quad \rightarrow \quad b_{a}=-\tau_{0 a}
$$

This constraint has two effects. First of all, it fixes $d-1 K$-transformations, as can be seen from the following transformation rule:

$$
\delta \mathcal{R}_{0 a}(H)=2 \lambda^{b} \tau_{a b}-\lambda^{b}{ }_{a} \mathcal{R}_{0 b}(H)-\lambda_{D} \mathcal{R}_{0 a}(H)+2 \lambda_{K a} .
$$

Note that this gauge-fixing leads to the following compensation transformation:

$$
\lambda_{K a}^{\mathrm{comp}}=-\lambda^{b} \tau_{a b} .
$$

At the same time, the gauge-fixing constraint (5.14) is a conventional constraint that allows us to solve for the spatial components of the dilatation gauge field as we did in the previous section. It is straightforward to check that after imposing the additional gauge-fixing condition (5.14) and identifying $\zeta^{v}=-\sigma, \lambda_{K}=\lambda_{K}^{+}$we obtain precisely the transformation rules (4.2) of $z=2$ Schrödinger geometry as obtained in the previous section.

For completeness we also give the transformation rules of the projective inverses:

$$
\begin{aligned}
\delta e^{\mu}{ }_{a} & =-\lambda_{a}^{b} e_{b}^{\mu}-\lambda_{D} e^{\mu}{ }_{a}, \\
\delta \tau^{\mu} & =-\lambda^{a} e^{\mu}{ }_{a}-2 \lambda_{D} \tau^{\mu} .
\end{aligned}
$$

We now consider the null-reduction of the dependent spin-connection and special conformal boost gauge fields. The reduction of the spin-connection components is very similar to the NC case. We find that the non-vanishing components are given by

$$
\begin{aligned}
\hat{\omega}_{\mu}^{a b}(\hat{e}, \hat{b}) & \equiv \omega_{\mu}^{a b}(e, \tau, m, b)=\stackrel{\leftrightarrow}{\omega}_{\mu}^{a b}(e, \tau, m, b)-m_{\mu} \tau^{a b}, \\
\hat{\omega}_{\mu}{ }^{a+}(\hat{e}, \hat{b}) & \equiv \omega_{\mu}{ }^{a}(e, \tau, m, b)=\dot{\circ}_{\mu}^{a}(e, \tau, m, b), \\
\hat{\omega}_{\mu}{ }^{a-}(\hat{e}, \hat{b}) & =e_{\mu b} \tau^{a b} \\
\hat{\omega}_{\mu}{ }^{-+}(\hat{e}, \hat{b}) & =b_{\mu}, \\
\hat{\omega}_{v}{ }^{a b}(\hat{e}, \hat{b}) & =\tau^{a b},
\end{aligned}
$$


where $\dot{\circ}_{\mu}^{a b}(e, \tau, m, b)$ and $\dot{\circ}_{\mu}^{a}(e, \tau, m, b)$ are the torsionless Schrödinger spin-connections, whose explicit expressions are given in eqs. (4.8) and (4.9), respectively.

Next, we consider the null-reduction of the gauge field of special conformal transformations $\hat{f}_{M}{ }^{A}$, defined in eq. (5.5). After a straightforward calculation we find the following expressions:

$$
\begin{aligned}
\hat{f}_{\mu}{ }^{a} & =-\frac{1}{d-1}\left(\mathcal{R}_{\mu b}\left(J^{a b}\right)+m_{\mu} \mathcal{D}_{b} \tau^{a b}-e_{\mu b} \mathcal{D}_{0} \tau^{a b}-\frac{1}{2 d} e_{\mu}{ }^{a} \mathcal{R}_{b c}\left(J^{b c}\right)\right)+\dot{\omega}_{\mu}{ }^{b} \tau_{b a} \\
\hat{f}_{\mu}{ }^{+} & =\frac{1}{d-1}\left(\mathcal{R}_{\mu a}^{\prime}\left(G^{a}\right)+\mathcal{R}_{\mu 0}^{\prime}(D)\right)-\frac{1}{2 d(d-1)} m_{\mu} \mathcal{R}_{a b}\left(J^{a b}\right) \\
\hat{f}_{\mu}{ }^{-} & =\frac{1}{2 d(d-1)} \tau_{\mu} \mathcal{R}_{a b}\left(J^{a b}\right)-\frac{1}{d-1} e_{\mu b} \mathcal{D}_{a} \tau^{a b}-\frac{1}{d-1} m_{\mu} \tau^{a b} \tau_{a b}, \\
\hat{f}_{v}{ }^{a} & =\frac{1}{d-1} \mathcal{D}_{b} \tau^{a b} \\
\hat{f}_{v}{ }^{+} & =\frac{1}{2 d(d-1)} \mathcal{R}_{a b}\left(J^{a b}\right) \\
\hat{f}_{v}{ }^{-} & =\frac{1}{d-1} \tau_{a b} \tau^{a b}
\end{aligned}
$$

where the gauge field $\hat{f}_{\mu}+$ is identified as the single gauge field $f_{\mu}$ of the reduced theory. The covariant derivative $\mathcal{D}$ is defined exactly as in (3.18), but this time with the spin-connections $\stackrel{\circ}{\omega}_{\mu}^{a b}(e, \tau, m, b)$ and $\stackrel{\circ}{\omega}_{\mu}^{a}(e, \tau, m, b)$, see eqs. (4.8) and (4.9). We observe that the component $\hat{f}_{\mu}{ }^{a}$ contains a torsion term with an explicit appearance of the spin-connection $\dot{\circ}_{\mu}{ }^{a}$. This is explained by the fact that $\hat{f}_{\mu}^{a}$ originally was a special conformal gauge field transforming as $\partial_{\mu} \lambda_{K}^{a}$ under the special conformal transformations. However, due to the gauge-fixing of those transformations, and in particular due to the compensating transformation given in eq. (5.16), we obtain $\delta \hat{f}_{\mu}^{a}=\partial_{\mu} \lambda^{b} \tau_{b a}+\ldots$, which explains the last term in eq. (5.24).

\section{$6 \quad$ NC gravity with arbitrary torsion}

In this section we will use our results on Schrödinger geometry with arbitrary torsion, derived in the previous section, to construct the $\mathrm{NC}$ gravity equations of motion for arbitrary torsion by applying the so-called conformal technique for the non-relativistic case [14]. We will give complete results for $d=3$ only.

It turns out that only the NC equations of motion with zero torsion $\left(\tau_{0 a}=0, \tau_{a b}=0\right)$ and with half-zero torsion $\left(\tau_{0 a}=0, \tau_{a b} \neq 0\right)$ are invariant under central charge transformations. However, the null-reduction by construction always leads to an answer that is invariant under central charge transformations. That is why we found that the onshell null-reduction of the Einstein equations leads to NC gravity with zero torsion. The half-zero torsion condition, although consistent with invariance under central charge transformations, has no clear causal structure and, as we saw above, does not follow from a null-reduction of General Relativity.

Applying the non-relativistic conformal technique [14], invariance under central charge transformations implies that we only need to introduce a real compensating scalar $\varphi$ for 
dilatations and not a second one to compensate for the central charge transformations. As was shown in [14], the SFT for this real scalar is given by ${ }^{10}$

$$
\text { SFT1 : } \quad \partial_{0} \partial_{0} \varphi=0, \quad \partial_{a} \varphi=0
$$

where the constraint $\partial_{a} \varphi$ is a consequence of the torsion condition $\tau_{0 a}=0$. In the absence of this torsion condition, the equation $\partial_{0} \partial_{0} \varphi=0$ is not invariant under Galilean boosts. To make this equation invariant under Galilean boosts, we introduce a second compensating scalar $\chi$ for central charge transformations. The important point is that under rigid Galilean boosts the spatial derivative of this compensating scalar $\chi$ transforms as [14]

$$
\delta\left(\partial_{a} \chi\right)=-\mathrm{M} \lambda_{a}
$$

where M is a mass parameter. Therefore, the lack of Galilean boost invariance of SFT1, see eq. (6.1), in the absence of the constraint $\partial_{a} \varphi=0$ can be compensated by adding further terms to this equation containing $\partial_{a} \chi$. In this way one ends up with the following SFT [14]:

$$
\text { SFT2 : } \quad \partial_{0} \partial_{0} \varphi-\frac{2}{\mathrm{M}}\left(\partial_{0} \partial_{a} \varphi\right) \partial_{a} \chi+\frac{1}{\mathrm{M}^{2}}\left(\partial_{a} \partial_{b} \varphi\right) \partial_{a} \chi \partial_{b} \chi=0 .
$$

The second compensating scalar breaks the invariance under central charge transformations. The SFT2 theory corresponds to either the twistless-torsional case $\left(\tau_{0 a} \neq 0, \tau_{a b}=0\right)$ or the arbitrary torsion case $\left(\tau_{0 a} \neq 0, \tau_{a b} \neq 0\right)$ case.

In a next step, we couple this SFT2 theory to the $z=2$ Schrödinger geometry with arbitrary torsion, we constructed in the previous section, by replacing all derivatives in (6.3) by Schrödinger covariant ones. In order to do this, it proves convenient to use a definition of the dependent gauge field $f_{\mu}$ of special conformal transformations that differs from the one given in eq. (4.13), by terms that transform covariantly under gauge transformations. In order to avoid confusion, we will denote this dependent gauge field by $F_{\mu}$. It is defined as the solution of the following conventional constraints

$$
\begin{aligned}
\mathcal{R}_{0 a}\left(G^{a}\right)-\frac{2}{\mathrm{M}}\left(D^{b} \chi\right) \mathcal{R}_{0 a}\left(J^{a}{ }_{b}\right)+\frac{1}{\mathrm{M}^{2}}\left(D^{b} \chi\right)\left(D^{c} \chi\right) \mathcal{R}_{c a}\left(J^{a}{ }_{b}\right) & \\
+\frac{1}{\mathrm{M}^{3}}\left(D^{b} \chi\right)\left(D_{b} \chi\right)\left(D^{c} \chi\right) D^{a} \tau_{c a} & =0, \\
\mathcal{R}_{0 a}(D) & =0
\end{aligned}
$$

where the curvatures are given by the expressions in eq. (4.12), with $f_{\mu}$ replaced by $F_{\mu}$. In particular one finds that $F_{0}=\tau^{\mu} F_{\mu}$ is given by

$$
\begin{aligned}
F_{0}=\frac{1}{d-1} & \left(\mathcal{R}_{0 a}^{\prime}\left(G^{a}\right)-\frac{2}{\mathrm{M}}\left(D^{b} \chi\right) \mathcal{R}_{0 a}\left(J^{a}{ }_{b}\right)+\frac{1}{\mathrm{M}^{2}}\left(D^{b} \chi\right)\left(D^{c} \chi\right) \mathcal{R}_{c a}\left(J^{a}{ }_{b}\right)+\right. \\
& \left.+\frac{1}{\mathrm{M}^{3}}\left(D^{b} \chi\right)\left(D_{b} \chi\right)\left(D^{c} \chi\right) D^{a} \tau_{c a}\right) .
\end{aligned}
$$

\footnotetext{
${ }^{10}$ By a Schrödinger Field Theory (SFT) we mean a field theory that is invariant under the rigid Schrödinger symmetries, see, e.g., [14].
} 
With this definition, $F_{\mu}$ transforms as follows under the different gauge transformations:

$$
\begin{aligned}
\tau^{\mu} \delta F_{\mu}= & \tau^{\mu}\left(\partial_{\mu} \lambda_{K}+2 \lambda_{K} b_{\mu}-2 \lambda_{D} f_{\mu}+2 \lambda^{b} \stackrel{\omega}{\omega}_{\mu}^{c} \tau_{b c}\right)-\frac{3}{\mathrm{M}} \lambda^{a}\left(D^{b} \chi\right) D_{0} \tau_{a b}+ \\
& +\frac{1}{\mathrm{M}^{2}} \lambda^{a}\left(D^{b} \chi\right)\left(D^{c} \chi\right) D_{c} \tau_{a b} \\
e^{\mu}{ }_{a} \delta F_{\mu}= & e^{\mu}{ }_{a}\left(\partial_{\mu} \lambda_{K}+2 \lambda_{K} b_{\mu}-2 \lambda_{D} f_{\mu}+2 \lambda^{b} \stackrel{\circ}{\omega}_{\mu}{ }^{c} \tau_{b c}\right)-2 \lambda^{b} D_{0} \tau_{b a} .
\end{aligned}
$$

The first step in coupling the equations of the SFT2 theory (6.3) to the $z=2$ Schrödinger geometry with arbitrary torsion consists of replacing all derivatives in the left-hand-side of (6.3) by Schrödinger covariant ones. This leads to the expression

$$
D_{0} D_{0} \varphi-\frac{2}{\mathrm{M}}\left(D_{0} D_{a} \varphi\right) D_{a} \chi+\frac{1}{\mathrm{M}^{2}}\left(D_{a} D_{b} \varphi\right) D_{a} \chi D_{b} \chi
$$

where the covariant derivatives are given by ${ }^{11}$

$$
\begin{aligned}
D_{0} D_{0} \varphi & =\tau^{\mu}\left(\partial_{\mu} D_{0} \varphi+b_{\mu} D_{0} \varphi+\stackrel{\circ}{\omega}_{\mu}^{a} D_{a} \varphi+F_{\mu} \varphi\right), \\
D_{0} D_{a} \varphi & =\tau^{\mu}\left(\partial_{\mu} D_{a} \varphi-\dot{\omega}_{\mu a}^{b} D_{b} \varphi+\dot{\circ}_{\mu}{ }^{b} \tau_{b a} \varphi\right), \\
D_{a} D_{b} \varphi & =e_{a}^{\mu}\left(\partial_{\mu} D_{b} \varphi-\stackrel{\circ}{\omega}_{\mu b}{ }^{c} D_{c} \varphi+\dot{\omega}_{\mu}{ }^{c} \tau_{c b} \varphi\right), \\
D_{0} \varphi & =\tau^{\mu}\left(\partial_{\mu}-b_{\mu}\right) \varphi, \quad D_{a} \varphi=e_{a}{ }^{\mu}\left(\partial_{\mu}-b_{\mu}\right) \varphi, \\
D_{a} \chi & =e_{a}^{\mu}\left(\partial_{\mu} \chi-\mathrm{M} m_{\mu}\right) .
\end{aligned}
$$

Note that the second covariant time derivative $D_{0} D_{0} \varphi$ of $\varphi$ contains the time-component $\tau^{\mu} F_{\mu}$ of the dependent special conformal gauge field gauge field $F_{\mu}$ given as a solution of eqs. (6.4).

The expression (6.7) can not be used yet as the starting point for defining a Schrödinger covariant equation, as it is not yet invariant under local boost transformations. Indeed, one finds that its variation under boosts is given by

$$
-\frac{2}{\mathrm{M}^{2}}\left(D^{a} \chi\right)\left(D^{b} \chi\right) \lambda_{a} \tau_{b}^{c} D_{c} \varphi-\frac{1}{\mathrm{M}^{2}}\left(D^{a} \chi\right)\left(D_{a} \chi\right) \lambda^{b} \tau_{b}^{c} D_{c} \varphi .
$$

We expect that this variation can be cancelled by adding further terms to the expression (6.7) via an iterative procedure but we did not yet find a closed answer in arbitrary dimensions. However, for the special case of $d=3$, the calculation simplifies significantly and the variation (6.13) can be cancelled by adding two extra terms to the expression (6.7). As a result, we find that the following equation is Schrödinger invariant in $d=3$ :

$$
\begin{aligned}
& D_{0} D_{0} \varphi-\frac{2}{\mathrm{M}}\left(D_{0} D_{a} \varphi\right)\left(D^{a} \chi\right)+\frac{1}{\mathrm{M}^{2}}\left(D_{a} D_{b} \varphi\right)\left(D^{a} \chi\right)\left(D^{b} \chi\right)- \\
& \quad-\frac{1}{\mathrm{M}^{3}}\left(D^{a} \chi\right)\left(D_{a} \chi\right)\left(D^{b} \chi\right) \tau_{b}^{c} D_{c} \varphi+\frac{1}{4 \mathrm{M}^{4}}\left(D^{a} \chi\right)\left(D_{a} \chi\right)\left(D^{b} \chi\right)\left(D^{c} \chi\right) \tau_{b}{ }^{d} \tau_{c d} \varphi=0 .
\end{aligned}
$$

To present the field equations, it is convenient to introduce the following boost invariant connection for spatial rotations

$$
\Omega_{\mu}{ }^{a b}=\dot{\omega}_{\mu}^{a b}+H_{\mu}{ }^{a b},
$$

\footnotetext{
${ }^{11}$ For the special case $\tau_{a b}=0$ the expressions were already given in [14].
} 
with the covariant tensor $H_{\mu}{ }^{a b}$ given by

$$
H_{\mu}^{a b}=\frac{1}{\mathrm{M}} D_{\mu} \chi \tau^{a b}+\frac{2}{\mathrm{M}}\left(e_{\mu}^{c} D^{[a} \chi+D^{c} \chi e_{\mu}{ }^{[a}\right) \tau_{c}^{b]}-\frac{2}{\mathrm{M}^{2}} \tau_{\mu} D_{c} \chi D^{[a} \chi \tau^{b] c} .
$$

With this definition, the curvature tensor

$$
\mathcal{R}_{\mu \nu}^{a b}(\Omega)=2 \partial_{[\mu} \Omega_{\nu]}^{a b}-2 \Omega_{[\mu}^{a c} \Omega_{\nu] c}^{b}
$$

is boost invariant and related to $\mathcal{R}_{\mu \nu}{ }^{a b}(J)$ via

$$
\mathcal{R}_{\mu \nu}^{a b}(\Omega)=\mathcal{R}_{\mu \nu}^{a b}(J)+2 D_{[\mu} H_{\nu]}^{a b}-2 H_{[\mu}^{a c} H_{\nu] c}^{b} .
$$

Since $\mathcal{R}_{\mu \nu}{ }^{a b}(\Omega)$ is boost invariant, one can consistently impose

$$
\mathcal{R}_{0 b}{ }^{b a}(\Omega)=0, \quad \mathcal{R}_{a c}{ }^{c b}(\Omega)=0
$$

as two of the NC field equations. Under boost transformations, the first equation in (6.19) transforms to the second one while the second one is invariant. These two equations are the extension to arbitrary torsion of the last two zero torsion NC equations given in eq. (3.22). The extension to arbitrary torsion of the first zero torsion equation $R_{0 a}\left(G^{a}\right)=0$ given in (3.22) can be found by imposing in eq. (6.14) the gauge-fixing conditions

$$
\varphi=1, \quad \chi=0,
$$

fixing the dilatations and central charge transformations, respectively. After substituting the expressions of the dependent Schrödinger gauge fields we derived in the previous two sections and using the other two torsional equations of motion (6.19), we find that this third torsional $\mathrm{NC}$ equation is given by

$$
\begin{array}{r}
\frac{1}{2} \mathcal{R}_{0 a}^{\prime}\left(G^{a}\right)-\tau^{\mu} \stackrel{\circ}{\omega}_{\mu}^{a} b_{a}-2\left(\mathcal{D}_{0} b_{a}\right) m^{a}-\left(\mathcal{D}_{a} b_{b}\right) m^{a} m^{b}-\left(b_{c}-\frac{1}{4} m^{c} \tau_{c d}\right) \tau^{b}{ }_{c} m^{a} m_{a} m^{b} \\
-\frac{1}{2} m^{a} m_{a} m^{b} D_{c} \tau_{b}{ }^{c}-m^{b} e_{a}{ }^{\nu}\left(\tau^{\mu}+\frac{1}{2} m^{c} e_{c}{ }^{\mu}\right)\left(2 D_{[\mu} H_{\nu]}{ }^{a b}-2 H_{[\mu}{ }^{a c} H_{\nu] c}{ }^{b}\right)=0
\end{array}
$$

where

$$
\mathcal{D}_{\mu} b_{a}=\partial_{\mu} b_{a}-\dot{\circ}_{\mu a}^{b} b_{b}+\dot{\circ}_{\mu}^{b} \tau_{a b},
$$

$b_{0}$ is gauge-fixed to zero, and, after the gauge-fixing (6.20), $H_{\mu}{ }^{a b}$ is given by

$$
H_{\mu}^{a b}=-m_{\mu} \tau^{a b}-2\left(e_{\mu}^{c} m^{[a}+m^{c} e_{\mu}^{[a}\right) \tau_{c}^{b]}-2 \tau_{\mu} m_{c} m^{[a} \tau^{b] c} .
$$

Note that $H_{\mu}{ }^{a b}$ vanishes identically for the special case that the torsion $\tau_{a b}$ is zero.

This finishes our discussion of NC gravity with arbitrary torsion in three dimensions whose equations can be found in eqs. (6.19) and (6.21). 


\section{Conclusions}

In this paper we applied two complementary techniques, gauging and null-reduction, to construct Newton-Cartan geometry and its conformal extension, $z=2$ Schrödinger geometry, with arbitrary torsion. The gauging technique has the advantage that it makes the symmetries resulting from the construction manifest. The null-reduction technique has the advantage that the construction is algorithmic and can easily be generalized to other cases as well. We explained why the null-reduction technique does not yield NC gravity with arbitrary torsion and showed, in three space-time dimensions, how equations of motion with arbitrary torsion can be obtained by applying the non-relativistic conformal method [14] using a SFT with two real compensating scalars: one compensating scalar $\varphi$ for the dilatations and one compensating scalar $\chi$ for the central charge transformations. This compensating technique leads to one of the equations of motion of torsional NC gravity, see eq. (6.21). This singlet equation is the one that contains the Poisson equation of the Newton potential. The other two equations, see eq. (6.19), followed by formulating them in terms of the curvature of a boost-invariant connection.

It would be interesting to extend the results of this paper to the supersymmetric case and apply the null-reduction technique to supergravity theories. The case of $d=3$ should lead to a generalization of the off-shell $3 \mathrm{D}$ NC supergravity constructed in $[17,18]$ to the case of arbitrary torsion. More interestingly, one can also take $d=4$ and construct 4D NC supergravity thereby obtaining, after gauge-fixing, the very first supersymmetric generalization of $4 \mathrm{D}$ Newtonian gravity. An intriguing feature of the $3 \mathrm{D}$ case is that the Newtonian supergravity theory contains both a Newton potential as well as a dual Newton potential. In analogy to the 3D case, we expect that in the supersymmetic case the Newton potential will not occur in the same representation as introduced by Newton. It would be interesting to see which representations of the Newton potential would occur in the 4D case and investigate whether this could have any physical effect.

\section{Acknowledgments}

E.A.B. and J.R. gratefully acknowledge support from the Simons Center for Geometry and Physics, Stony Brook University at which part of the research for this paper was performed during the workshop Applied Newton-Cartan Geometry. E.A.B. and J.R. also thank the Galileo Galilei Institute in Firenze for the stimulating atmosphere during the workshop Supergravity, what next?, where this work was initiated. E.A.B. wishes to thank the University of Vienna for its hospitality. The work of A.Ch. was supported by the H2020 Twinning project No. 692194, "RBI-T-WINNING".

Open Access. This article is distributed under the terms of the Creative Commons Attribution License (CC-BY 4.0), which permits any use, distribution and reproduction in any medium, provided the original author(s) and source are credited. 


\section{References}

[1] M.H. Christensen, J. Hartong, N.A. Obers and B. Rollier, Torsional Newton-Cartan geometry and Lifshitz holography, Phys. Rev. D 89 (2014) 061901 [arXiv:1311.4794] [INSPIRE].

[2] M. Geracie, D.T. Son, C. Wu and S.-F. Wu, Spacetime symmetries of the quantum Hall effect, Phys. Rev. D 91 (2015) 045030 [arXiv: 1407.1252] [INSPIRE].

[3] D. Van den Bleeken, Torsional Newton-Cartan gravity from the large c expansion of general relativity, Class. Quant. Grav. 34 (2017) 185004 [arXiv:1703.03459] [InSPIRE].

[4] J.M. Luttinger, Theory of thermal transport coefficients, Phys. Rev. 135 (1964) A1505 [INSPIRE].

[5] A. Gromov and A.G. Abanov, Thermal Hall effect and geometry with torsion, Phys. Rev. Lett. 114 (2015) 016802 [arXiv:1407.2908] [INSPIRE].

[6] M. Geracie, S. Golkar and M.M. Roberts, Hall viscosity, spin density and torsion, arXiv:1410.2574 [INSPIRE].

[7] M. Geracie, K. Prabhu and M.M. Roberts, Physical stress, mass and energy for non-relativistic matter, JHEP 06 (2017) 089 [arXiv:1609.06729] [INSPIRE].

[8] C. Duval, G. Burdet, H.P. Kunzle and M. Perrin, Bargmann structures and Newton-Cartan theory, Phys. Rev. D 31 (1985) 1841 [InSPIRE].

[9] B. Julia and H. Nicolai, Null Killing vector dimensional reduction and Galilean geometrodynamics, Nucl. Phys. B 439 (1995) 291 [hep-th/9412002] [INSPIRE].

[10] K. Jensen, On the coupling of Galilean-invariant field theories to curved spacetime, arXiv: 1408.6855 [INSPIRE].

[11] X. Bekaert and K. Morand, Connections and dynamical trajectories in generalised Newton-Cartan gravity I. An intrinsic view, J. Math. Phys. 57 (2016) 022507 [arXiv: 1412.8212] [INSPIRE].

[12] G. Festuccia, D. Hansen, J. Hartong and N.A. Obers, Torsional Newton-Cartan geometry from the Noether procedure, Phys. Rev. D 94 (2016) 105023 [arXiv:1607.01926] [INSPIRE].

[13] R. Andringa, E. Bergshoeff, S. Panda and M. de Roo, Newtonian gravity and the Bargmann algebra, Class. Quant. Grav. 28 (2011) 105011 [arXiv:1011.1145] [INSPIRE].

[14] H.R. Afshar, E.A. Bergshoeff, A. Mehra, P. Parekh and B. Rollier, A Schrödinger approach to Newton-Cartan and Hořava-Lifshitz gravities, JHEP 04 (2016) 145 [arXiv:1512.06277] [INSPIRE].

[15] E.A. Bergshoeff, J. Hartong and J. Rosseel, Torsional Newton-Cartan geometry and the Schrödinger algebra, Class. Quant. Grav. 32 (2015) 135017 [arXiv:1409.5555] [INSPIRE].

[16] J. Hartong and N.A. Obers, Hořava-Lifshitz gravity from dynamical Newton-Cartan geometry, JHEP 07 (2015) 155 [arXiv:1504.07461] [INSPIRE].

[17] E. Bergshoeff, J. Rosseel and T. Zojer, Newton-Cartan (super)gravity as a non-relativistic limit, Class. Quant. Grav. 32 (2015) 205003 [arXiv: 1505.02095] [INSPIRE].

[18] E. Bergshoeff, J. Rosseel and T. Zojer, Newton-Cartan supergravity with torsion and Schrödinger supergravity, JHEP 11 (2015) 180 [arXiv: 1509.04527] [INSPIRE]. 